\title{
Unsupervised Anomaly Detection for Communication Networks: an Autoencoder Approach
}

\author{
Pieter Bonte ${ }^{[0000-0002-8931-8343] 1}$, Sander Vanden Hautte ${ }^{10000-0002-5035-4419]}$, \\ Annelies Lejon ${ }^{2}$, Veerle Ledoux ${ }^{2}$, Filip De Turck ${ }^{10000-0003-4824-1199]}$, Sofie Van \\ Hoecke ${ }^{[0000-0002-7865-6793] 1}$, and Femke Ongenae ${ }^{1[0000-0003-2529-5477]}$ \\ 1 IDLab, Ghent University - imec \\ Technologiepark-Zwijnaarde 126 \\ B-9052, Gent, Belgium \\ pieter.bonte@ugent.be \\ 2 Skyline Communications \\ Ambachtenstraat 33 \\ 8870, Izegem, Belgium
}

\begin{abstract}
Communication networks are complex systems consisting of many components each producing a multitude of system metrics that can be monitored in real-time. Anomaly Detection (AD) allows to detect deviant behavior in these system metrics. However, in communication networks, large amounts of domain knowledge and huge manual efforts are required to efficiently monitor these complex systems. In this paper, we describe how AutoEncoders (AE) can elevate the manual effort for unsupervised $\mathrm{AD}$ in communication networks. We show that $\mathrm{AE}$ can be applied, without domain knowledge or manual effort and evaluate different types of $\mathrm{AE}$ architectures and how they perform on a variety of anomaly types found in communication networks.
\end{abstract}

Keywords: Anomaly detection - Time Series · Communication Networks AutoEncoders.

\section{Introduction}

Anomaly Detection (AD) in communication networks is a challenging task as these systems consist of many components that produce various real-time system metrics. The sheer scale and the dynamic nature of these systems make traditional AD inadequate as these techniques require knowledge about the domain, information about the environment the components are deployed in, or the actual configuration of the network and its components that are being used. As many traditional AD techniques are threshold-based, this becomes infeasible to manually configure and maintain thresholds for anomalies and leads to high rates of false alarms [4].

Skyline Communications ${ }^{3}$ monitors communication networks for various providers worldwide. The company provides solutions for monitoring the broadcast, satellite, cable, telco, and mobile industry. When the network is showing anomalous behavior, it needs to be detected early on, preventing communication downtime and financial

\footnotetext{
${ }^{3}$ https://skyline.be/
} 


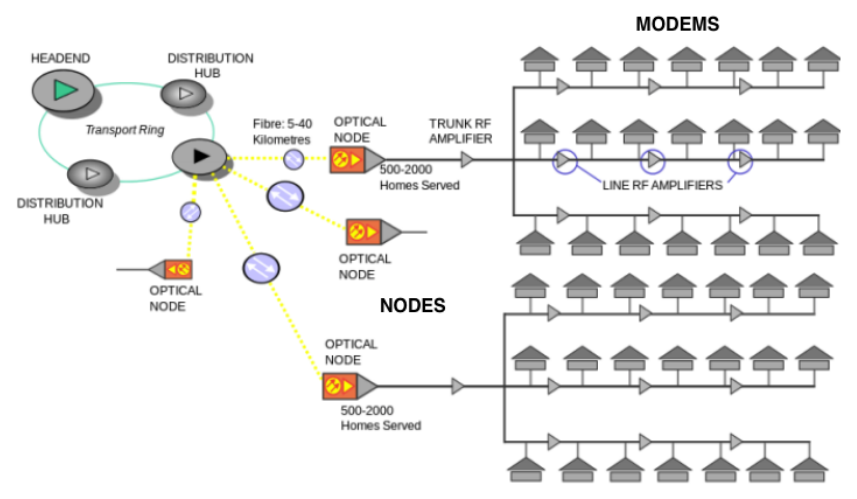

Fig. 1. Overview of the communication network use case: on the right we see various modems capturing system metrics regarding the state of the network. On the left, we see that multiple of these modems are connected to one node.

loss. Skyline employs AD to detect network failures early-on and informs its customers of potential system risks. However, these customers typically only want to be notified about anomalies having a major influence on the health of their systems. Being notified about non-critical anomalies is financially expensive due to the high maintenance costs. It is thus important to reduce the number of false positives, and to not overload the customers. Skyline also requires a generic approach, as each of their customers can have slightly different system metrics, different network configurations or completely different components. Furthermore, their customers typically do not want to share their data due to the sensitivity of the data. This requires the training and deployment of on-premise AD. As the models are trained and deployed on the hardware of the customer, these models should thus not require too much preprocessing. Most importantly, it is typically unknown in advance what could be considered an anomaly, requiring unsupervised approaches for detecting system failures.

In this paper, we aim to show how AutoEncoders (AE) can ideally be employed to perform $\mathrm{AD}$ in communication networks. We describe how AEs can learn normal behavior in communication networks, without the need for large amounts of preprocessing or domain expertise. We show how AE can predict anomalies when the behavior deviates from normal behavior. In the next section, we go deeper into the specific AD use case in communication networks and define the requirements for the system. We then describe related work and more details regarding AEs. We describe the differences between various $\mathrm{AE}$ architectures and evaluate their $\mathrm{AD}$ performance on both synthetic as real-life data.

\section{Use Case \& Requirements}

This Section describes the use case and requirements.

\subsection{Use case}

Figure 1 visualizes a typical communication network. On the right we see various modems positioned at certain households. These modems capture real-time system 
metrics, which are retrieved by Skyline. In the communication network, these modems are connected to an optical node, that takes care of serving up to 2000 households. Even though data is captured at modem-level, anomalies should be reported at node-level, as network providers are concerned with more global system failures. Each modem provides the following system metrics:

- status: the status of the modem, indicates if the modem is online or not.

- US Tx: the upstream transmit power of the received communication signal.

- SNR: the signal-to-noise ratio of the signal.

- CR: the corrected ratio that was necessary to correct the received data fragments.

- UR: the ration of uncorrected data fragments.

- CS: a metric indicating various timeouts.

- PreMTTER: total energy ratio measured before combining the signals of various modems.

- PostMTTER: total energy ratio measured after combining the signals of various modems.

The status metric is categorical, while the other metrics are continuous. It is important to note that these various systems metrics can be considered as data streams or timeseries and that they describe the metrics for their own system, i.e. the modem. As AD needs to be performed on a node-level, these metrics are aggregated. The aggregation is done by taking the mean of metrics of the available modems under each node. Note that some modems might be offline.

\subsection{Anomaly types}

In communication networks, four types of anomalies can occur, each type is visualized in Figure 2 and described below:

- Outliers: an abrupt spike of a certain signal, e.g. due to a temporal communication loss with a satellite.

- Trend changes: the signal (slowly) changes to a different kind of behavior, typically for a longer time period, e.g. due to wrongly configured modem.

- Variance changes: the variance of the signal changes, e.g. due to malfunctioning cable.

- Level shifts: the signal temporally takes a different kind of behavior, due to temporally downtime of upstream components.

We note that the trend changes and level shifts are depicted in Figure 2 in an increase of signal fashion, however, a decrease in signal is also possible.

\subsection{Requirements}

Based on the description of the use case and the different types of anomalies the following requirements can be extracted:

1. Unsupervised: as there are no labels available, the AD should be unsupervised.

2. Multi-variate: each modem is producing multiple data streams that might need to be correlated. 


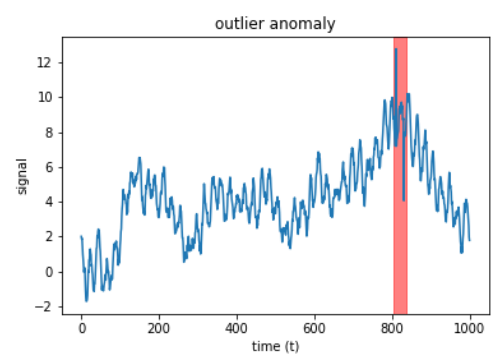

(a) Outlier anomaly

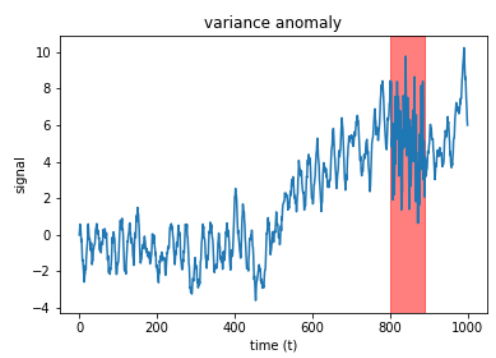

(c) Variance change anomaly

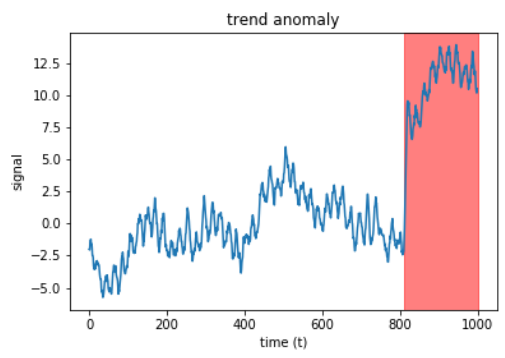

(b) Trend change anomaly

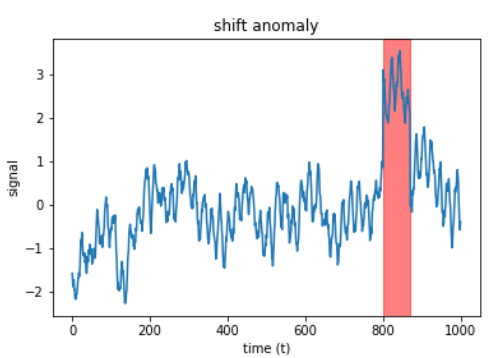

(d) Level shift anomaly

Fig. 2. The different type of communication network anomalies indicated in red.

3. Generic: applicable in many use cases without the need for much manual effort such as extensive preprocessing. This is important such that the same techniques can be used for different customers.

4. Accurate: as the number of false positives should be minimal.

5. Context-aware: to reduce the number of false positives, it should be possible to add additional context. For example, modems can have different hardware versions, resulting in different behavior. Taking this context into account allows to better differentiate the data.

6. Adaptable: as trends and seasonality in data might change, the AD should be able to adapt in order to reduce the number of false positives.

7. Efficiency: as the AD should happen on-premise, learned AD should be able to be transferred to other scenarios to reduce learning.

These requirements will allow us to narrow the search for applicable $\mathrm{AD}$ techniques.

\subsection{AD techniques}

Many techniques exist to perform $\mathrm{AD}$ over time-series data, some of the most prevalent examples are, autoregressive models such as ARIMA and all its variants [9], matrix profiles [12], AutoEncoders (AE) [13], clustering-based approaches, one-class classifications such as one-class SVM [8] or discrete-state models such as hidden Markov models [5]. However, when the requirements set above are mapped upon these techniques, many proposed methods fail one or more of these requirements. However, one that fulfills them all and appears promising here is the AutoEncoder. We give a brief overview of how $\mathrm{AE}$ fulfill the requirements: 
- Unsupervised: AE are by design unsupervised.

- Multi-variate: AE can take multiple inputs.

- Generic: AE can learn non-linear relations between features reducing the need for preprocessing. Furthermore, non-important input features are given lower weights and thus do not influence the model. Many statistical approaches require the removal of trends and seasonality, as preprocessing step. AE can cope with trend and seasonality and are more robust against noisy data.

- Context-aware: AE can take any kind of input and can extend the input with additional data.

- Adaptable: the weights of an AE can be updated with new data.

- Transferable: AE allow transfer learning by reusing some of the layers.

\section{Related Work}

There is abundant literature on the topic of AD. We focus on approaches related to AD for communication networks. Pawling et al. describe a one-pass clustering-based approach for $\mathrm{AD}$ in wireless communication networks [11]. It is an optimization of the k-means clustering algorithm that allows efficient updating of the clusters. However, the approach requires heavy preprocessing and there is no support to use easily transfer the models to other scenarios. Brutlag et al. propose an extension of Holt-Winters Forecasting, which supports incremental model updates through exponential smoothing [3], for detecting aberrant behavior in time series for network monitoring. Oki et al. propose a solution for detecting mobile network failure by combining both operator and social data [10]. They use an ensemble model consisting of logistic regression, random forest, and an AE. However, they assume a labeled dataset. Dornel et al. describe a solution for monitoring computer networks using traffic forcasting [6]. They employ Exponential Smoothing and ARIMA models to detect possible future abnormal behavior of network usage. Ageev et al. elaborate on a technique using fuzzy logics to detect abnormal traffic in IoT networks [1]. Liu et al. focus on a network monitoring platform that allows to easily label detected anomalies [7]. A random forest model is then used to classify the anomalies. However, the technique is not unsupervised and is thus out-of-scope for this research as there are no labels available.

In conclusion, previous research has mainly focused on fixed models for specific cases that typically only take into account data streams without any support for additional context data or easily using the models in different scenarios. We are interested in an approach that can be easily deployed in various scenarios and take additional context data into account to reduce false positives. We are the first to study how AEs can be utilized in communication networks for efficient AD.

\section{Autoencoders for Unsupervised Anomaly Detection}

In order to explain how we can use AEs for the generic processing of multivariate datastreams, we first define what a stream is:

Definition 1. A Stream $\mathscr{S}$ is a possible unbounded ordered sequence of data, i.e. $\mathscr{S}=\left\{s_{0}, s_{1}, s_{2}, \ldots\right\}$ with $s_{i}$ the data occurring at timestamp $i$. 


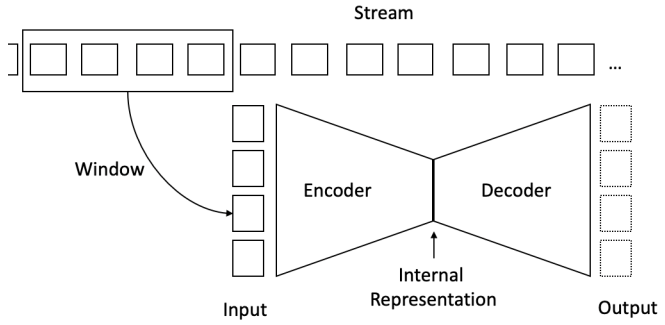

Fig. 3. Overview of the AE architecture. The encoder takes the windowed data stream and transforms it into the internal representation of lower dimension and the decoder predicts the input again from this internal representation.

As it is not possible to process unbounded streams of data (as it has no end), we define a time-based windowing function that can chunk the stream in processable parts:

Definition 2. A (time-based) window function $\mathscr{W}_{i}$ over a Stream $\mathscr{S}$, is a function that splits the stream in processable chunks of data of size $i$. $\mathscr{W}_{i}(\mathscr{S})=\left\{\left(s_{0}, . ., s_{i}\right),\left(s_{i+1}, . ., s_{2 i}\right), \ldots\left(s_{j}, . . s_{j+i}\right), \ldots\right\}$.

An AE is a neural network that learns to reconstruct its input by enforcing a bottleneck on the dimension of the latent space that represents the compressed representation of the data. Figure 3 shows how the AE takes the windowed data stream as input and converts it to an internal representation of a lower dimension. The decoder then reconstructs the input from this internal representation. As the internal representation is a bottleneck in the network, it needs to summarize the most important characteristics. Thus an $\mathrm{AE}$ can be seen as a function that reconstructs its input from the input itself, i.e. $f_{a e}(x)=x^{\prime}$ while reducing the internal representation in $f_{a e}$.

Definition 3. The reconstruction error (RE) calculates how different the reconstructed input is from the input itself, i.e. $d\left(x, x^{\prime}\right)$ with $d$ a distance function.

An anomaly can be flagged when the RE is above a certain threshold. In AD, an AE can learn the normal behavior of the data stream, as it represents the stream characteristics in its internal representation. This assumption is valid when one does feed normal data, i.e. data without anomalies, to the network. As it is often not possible to know what normal behavior is, the assumption still holds when the dataset is unbalanced, i.e. the number of anomalies is much lower than the normal data.

Many different architectures of AE exist, we list some of the most prominent configurations:

- Dense AE: a neural network with at least three dense layers, where the hidden layers decrease in size on the encoder part and increase in size on the decoder part, enabling a bottleneck in the middle hidden layer. Figure 3 visualizes the Dense AE.

- LSTM AE: uses one or more LSTMs to encode the signal to an internal representation and uses a dense layer to decode the internal representation.

- LSTM seq2seq: uses one or more LSTMs to encode the signal to an internal representation and uses one or more LSTMs to decode the internal representation.

- TCN AE: similar to the LSTM AE, but uses a Temporal Convolutional Network (TCN) [2] for encoding the time series instead of the LSTM cell. 
- TCN seq2seq: similar to the LSTM seq2seq, but uses a TCN [2] for encoding instead of the LSTM. An LSTM is still used for decoding as there is no upsampling variant for the temporal convolutions of the TCN.

In the next section, we describe how the different architectures compare and how AEs, in general, can deal with the set requirements.

\section{Evaluation}

In the next section, we investigate which of the architectures can ideally be used for $\mathrm{AD}$ in communication networks and how well AEs meet the set requirements from Section 2.3. First we evaluate the different AE architectures on generated data and then we evaluate on the Skyline use case.

\subsection{AE architecture evaluation}

First, we will evaluate if the different types of $\mathrm{AE}$ architectures are able to detect the variety of anomaly types described in Section 2.2. The time series for this evaluation and their anomalies were generated using the time series anomaly generator AGOTS 4 . We have trained each $\mathrm{AE}$ architecture on a series of 1000 samples without anomalies and evaluated on a modified version of the series that includes the anomalies.

We used a window of size 10 and 100 epochs to train each architecture. First, we visually show the behavior of the different architectures for the selected anomalies from Section 2.2. We evaluated this behavior over more than 30 different generated time series for each anomaly type and verified the visualized behavior of each architecture depicted below. Table 1 also summarizes the averaged precision-recall characteristics, using the average area under the curve (AUC) of the precision-recall curve. These curves calculate the precision and recall when employing different thresholds on the REs in order to flag an anomaly. For each anomaly type, we first show the RE of each architecture. In order to detect the anomaly, the RE should visually peak on the location of the anomaly. This means that the RE is higher for the anomalous behavior, indicating that the AE had difficulties reconstructing the signal, as it is not part of the learnt normal behavior.

We now discuss how the different architecture perform on the different AD types: Outlier Anomalies: Figure 4 depicts the REs for each architecture on the outlier anomaly. We see that all of the architectures reasonably detect the outliers.

Trend Anomalies: Figure 5 depicts the REs for each architecture on the outlier anomaly. We see that the dense AE produces a spike when at the changing point, however, fails to detect that the trend has changed. We see that the seq2seq approaches are more robust to changes in the normal signal, even though they have generally a much higher RE, even for the normal behavior. The LSTM AE and TCN AE are more prone to show higher REs due to high values in the input signal.

Variance Anomalies: Figure 6 shows the results for the variance anomly type. We can see that all architectures produce reasonable results.

Shift Anomalies: Figure 7 shows the results for the shift anomaly type. All architectures detect the shift, however, the dense AE only detects the outer changes. This can

\footnotetext{
${ }^{4}$ https://github.com/KDD-OpenSource/agots
} 


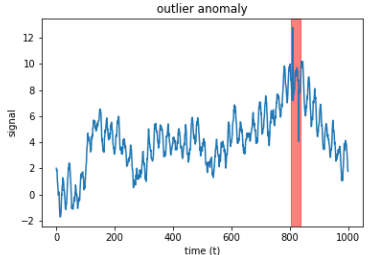

(a) Outlier anomaly

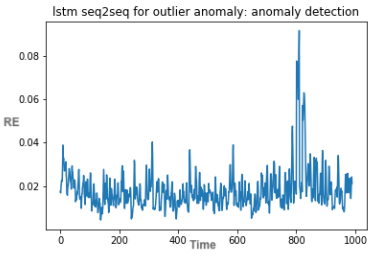

(d) LSTM seq2seq

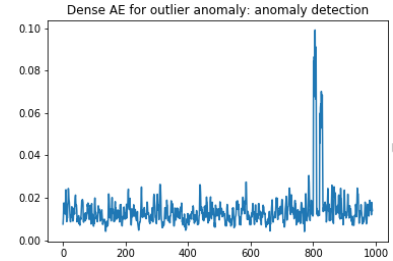

(b) Dense AE

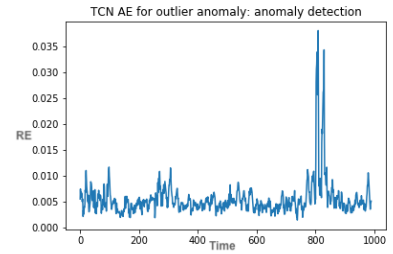

(e) TCN AE

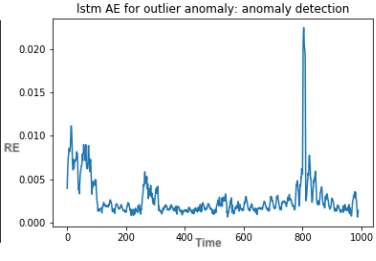

(c) LSTM AE

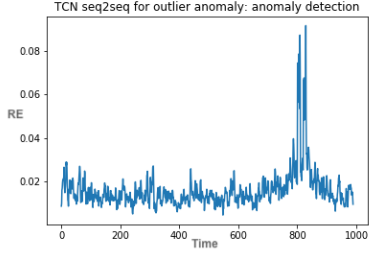

(f) $\mathrm{TCN}$ seq $2 \mathrm{seq}$

Fig. 4. Visualization of the RE for outlier anomaly.

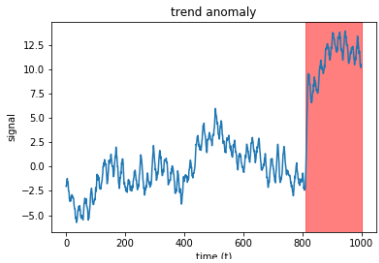

(a) Trend anomaly

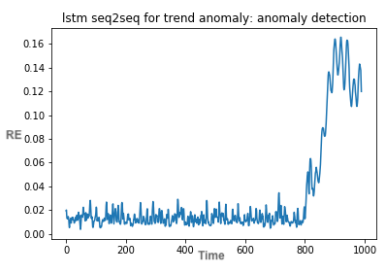

(d) LSTM seq2seq

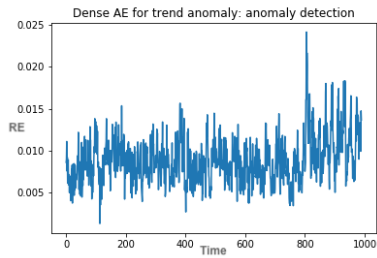

(b) Dense AE

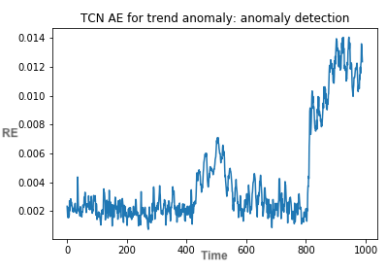

(e) TCN AE

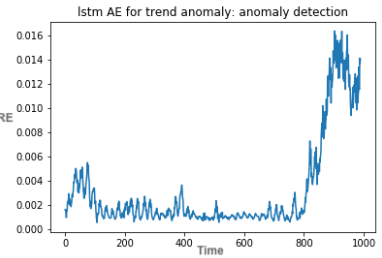

(c) LSTM AE

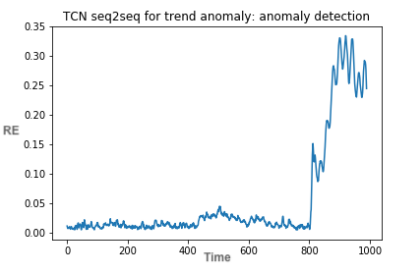

(f) $\mathrm{TCN}$ seq $2 \mathrm{seq}$

Fig. 5. Visualization of the RE for trend anomaly. 
Generic I Insunervised Anomalv Detection

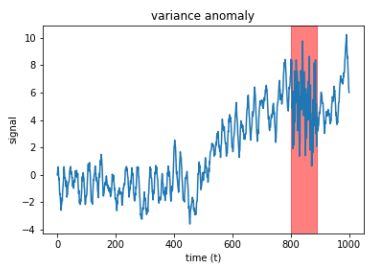

(a) Variance Anomaly

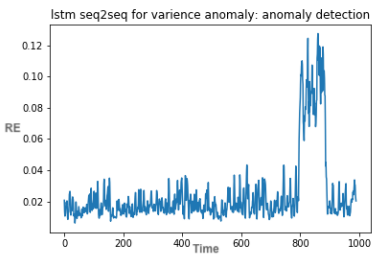

(d) LSTM seq2seq

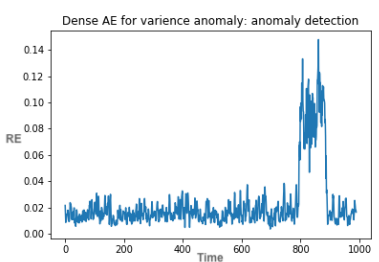

(b) Dense AE

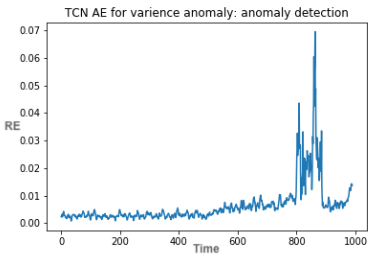

(e) TCN AE

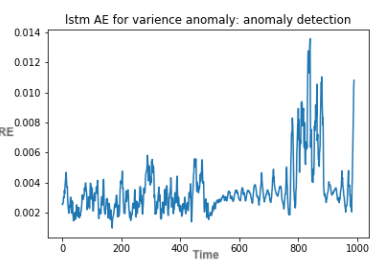

(c) LSTM AE

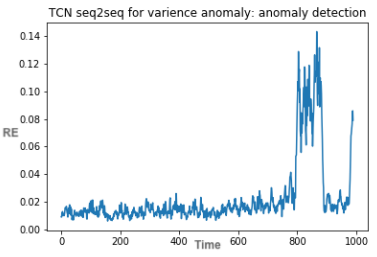

(f) $\mathrm{TCN}$ seq $2 \mathrm{seq}$

Fig. 6. Visualization of the RE for variance anomaly.

be seen as the RE during the shift drops again. The other architectures are less prone to this behavior. We see that the LSTM AE is again reacting to the subtle changes in normal behavior.

We have shown the performance for the different architectures on 4 types of anomalies. The dense AE can really well detect abrupt changes in signal behavior, however, fails to more long term changes. The LSTM AE can reconstruct the signal really good, however, it is prone to subtle changes in the normal behavior of the signal, causing it to produce false positives. The TCN AE shows similar behavior, however, more subtle. This is because, compared to the dense and seq2seq approaches, the LSTM and TCN AE have a limited bottleneck. The LSTM seq2seq is more robust even with higher REs. However, it is still noisier compared to the TCN seq2seq approach. The TCN seq2seq approach produces the least variance on the reconstruction of the normal behavior while clearly detecting all anomaly types.

Table 1 summarizes these findings and reports the average AUC for the different evaluations for each architecture. We see that overall the TCN seq2seq performs best, even though its RE is higher for the normal behavior than the other approaches.

Table 1. Comparison the the different AE architectures. ('+': detects the anomalies, '-': does not detect or only partly detects the anomalies; $\mathrm{RE}=$ Reconstruction Error.)

\begin{tabular}{|l|c|c|c|c|c|c|c|c|c|c|}
\cline { 2 - 12 } \multicolumn{1}{c|}{} & \multicolumn{2}{l|}{ Outliers } & \multicolumn{2}{l|}{ Trend } & \multicolumn{2}{l|}{ Variance } & \multicolumn{2}{l|}{ Shift } & $\begin{array}{c}\text { low variance } \\
\text { normal behaviour }\end{array}$ & $\begin{array}{c}\text { low RE } \\
\text { normal behaviour }\end{array}$ \\
\cline { 2 - 12 } & score & $\begin{array}{l}\text { avg } \\
\text { AUC }\end{array}$ & score & $\begin{array}{l}\text { avg } \\
\text { AUC }\end{array}$ & score & $\begin{array}{l}\text { avg } \\
\text { AUC }\end{array}$ & score & $\begin{array}{l}\text { avg } \\
\text { AUC }\end{array}$ & & \\
\hline Dense AE & + & 0.86 & - & 0.39 & + & 0.98 & - & 0.43 & + & - \\
\hline LSTM AE & - & 0.29 & + & 0.81 & + & 0.65 & + & 0.65 & - & + \\
\hline LSTM seq2seq & + & 0.74 & + & 0.83 & + & 0.97 & + & 0.76 & + & + \\
\hline TCN AE & + & 0.53 & + & 0.81 & + & 0.74 & + & 0.72 & - & - \\
\hline TCN seq2seq & + & 0.80 & + & 0.86 & + & 0.97 & + & 0.81 & + & + \\
\hline
\end{tabular}




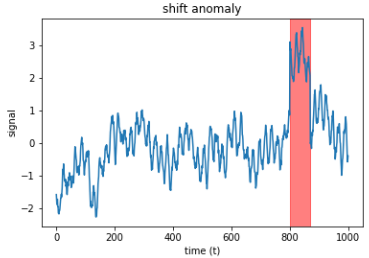

(a) Shift anomaly

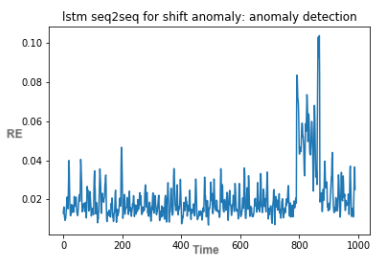

(d) LSTM seq2seq

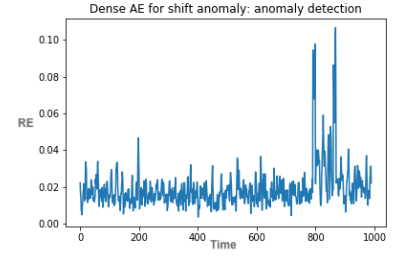

(b) Dense AE

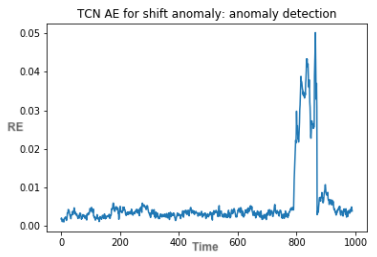

(e) TCN AE

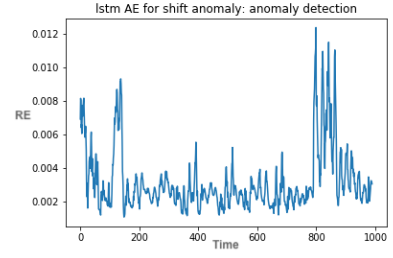

(c) LSTM AE

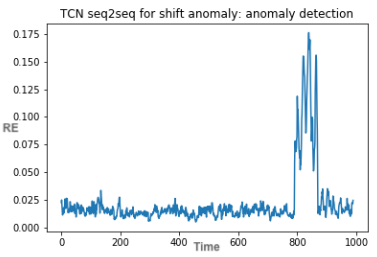

(f) $\mathrm{TCN}$ seq $2 \mathrm{seq}$

Fig. 7. Visualization of the RE for shift anomaly.

\subsection{Skyline use case evaluation}

As we operate in an unsupervised environment with no labels available, it is not trivial to evaluate the correctness of the anomaly detection approach. Skyline provided us with anomaly scores that were based on thorough investigation of the time series, heavily preprocessing and expert knowledge.

Dataset: We evaluated the data of 25 nodes. Each data stream describes the aggregated data from the underlying modems contained in 16 features. Each data stream contains 4000 samples, i.e. data over a period of two weeks at a frequency of 5 minutes. We used the first $60 \%$ of the time series to train upon and the remainder for validation and testing purposes.

Evaluation: We have performed hyper-parameter tuning for each node, optimizing the architecture, the number of used neurons, the size of the window, the optimizer, and the dropout rate. Table 2 shows the performance of the different AE architectures. On the left table, we report for a random selection of 10 nodes the architecture that achieved the best AUC scores for the Receiver Operator Curves (ROC). The ROC curve gives an indication of the true positives compared to the false positives when varying the thresholds on the REs. When using a very low threshold, all anomalies would be found, however, some normal events will be flagged as well. We see that the AUC values are pretty high, indicating that the selected models are doing a great job at identifying the anomalies, without triggering too many false positives. We do not achieve perfect scores, as the baseline we compare with is also not perfect. We have validated that we can detect all baseline anomalies, however, typically reporting more anomalies depending on the chosen threshold. Varying the threshold allows to differentiate between the severity of the detected anomalies.

Table 2 shows that different architectures are chosen for the different nodes and there is no clear winner. On the right of Table 2 we report the percentage each architecture is 
Table 2. Evaluation of the AE architectures on the skyline use case. Left the AUC of the ROC for a selection of 10 nodes. We report the best AUC for the best architecture. Right we report the percentages of nodes in the dataset each architecture performed best, with their average AUC.

\begin{tabular}{|c|c|c|}
\hline nodeID & AUC & architecture \\
\hline node1 & 0.75 & LSTM seq2seq \\
\hline node2 & 0.90 & LSTM AE \\
\hline node3 & 0.69 & TCN AE \\
\hline node4 & 0.99 & Dense AE \\
\hline node5 & 0.84 & TCN seq2seq \\
\hline node6 & 0.93 & LSTM seq2seq \\
\hline node7 & 0.90 & LSTM AE \\
\hline node8 & 0.97 & Dense AE \\
\hline node9 & 0.99 & Dense AE \\
\hline node10 & 0.94 & LSTM seq2seq \\
\hline $\begin{array}{c}\text { all } \\
\text { nodes }\end{array}$ & 0.81 & Dense AE \\
\hline
\end{tabular}

\begin{tabular}{|c|l|l|}
\hline architecture & $\begin{array}{l}\text { selection } \\
\text { percentage }\end{array}$ & $\begin{array}{l}\text { AVG } \\
\text { AUC }\end{array}$ \\
\hline Dense AE & $24 \%$ & 0.96 \\
\hline LSTM AE & $8 \%$ & 0.90 \\
\hline LSTM seq2seq & $28 \%$ & 0.90 \\
\hline TCN AE & $28 \%$ & 0.83 \\
\hline TCN seq2seq & $12 \%$ & 0.86 \\
\hline
\end{tabular}

chosen as the best model, together with the average AUC they report. We see that there is a tie between the Dense AE, LSTM seq2seq, and the TCN AE. However, in the cases that the Dense AE performed best, it also reports the highest achieved AUC values. From Table 1 we already showed that the Dense AE is good at detecting outliers and the skyline case contains many outlier anomalies. We also report the AUC for a model that has been trained on all the node training data and evaluated on their combined test sets. In this case, the Dense AE also performs best, surprisingly with a larger window. All models that were trained on a single node achieved the best performance using a window of 5, while the model for all nodes combined achieved the best results using a larger window of 10 . Furthermore, this Dense AE was a deeper model, i.e. 5 layers, compared to the shallow dense models with a single hidden layer that performed best for the single nodes. This can be explained by the fact that the model needs to extract many more different types of time series behavior over multiple nodes, requiring deeper models to extract and generalize these different behaviors. We can see that the AEs are able to detect the anomalies in complex communication networks without preprocessing, domain expertise or large manuel efforts. The only preprocessing required is differentiating between continuous and categorical features.

\section{Conclusion}

We have shown that AEs can detect anomalies in communication networks in a generic fashion, i.e. without the need for large amounts of preprocessing ${ }^{5}$, by learning the normal behavior of the time series. We have evaluated the performance of different $\mathrm{AE}$ architectures on 4 types of anomalies commonly found in communication networks. Furthermore, we have evaluated these architectures on a real communication network dataset. We showed that TCN seq 2 seq architectures perform best on the artificial datasets, however, the dense AE showed best results on the real use case data. In future work,

\footnotetext{
${ }^{5}$ We note that the technique does require some hyperparameter tuning.
} 
we will investigate the theoretical foundation of the different architectures to theoretically explain their behavior for different anomaly detection problems and investigate ensembles to combine their strengths. We will also investigate a method for automatically extracting the best threshold in an unsupervised manner. Furthermore, we will investigate the best ways to exploit context in AEs to further increase the accuracy.

Acknowledgement: This research was funded by the imec.icon project RADIANCE, which was co-financed by imec, VLAIO, Barco, ML6 and Skyline.

\section{References}

1. Ageev, S., et al.: Abnormal traffic detection in networks of the internet of things based on fuzzy logical inference. In: 2015 XVIII SCM. pp. 5-8. IEEE (2015)

2. Bai, S., et al.: An empirical evaluation of generic convolutional and recurrent networks for sequence modeling. arXiv preprint arXiv:1803.01271 (2018)

3. Brutlag, J.D.: Aberrant behavior detection in time series for network monitoring. In: LISA. vol. 14, pp. 139-146 (2000)

4. Chandola, V., et al.: Anomaly detection: A survey. ACM computing surveys (CSUR) 41(3), 15 (2009)

5. Cho, S.B., et al.: Efficient anomaly detection by modeling privilege flows using hidden markov model. computers \& security 22(1), 45-55 (2003)

6. Dornel, H., et al.: Traffic forecasting for monitoring in computer networks using time series. International Journal of Advanced Engineering Research and Science 6(7) (2019)

7. Liu, D., et al.: Opprentice: Towards practical and automatic anomaly detection through machine learning. In: Proceedings of the 2015 Internet Measurement Conference. pp. 211224. ACM (2015)

8. Manevitz, L.M., et al.: One-class svms for document classification. Journal of machine Learning research 2(Dec), 139-154 (2001)

9. Mehrotra, K.G., et al.: Anomaly detection principles and algorithms. Springer (2017)

10. Oki, M., et al.: Mobile network failure event detection and forecasting with multiple user activity data sets. In: Thirty-Second AAAI Conference on Artificial Intelligence (2018)

11. Pawling, A., et al.: Anomaly detection in a mobile communication network. Computational and Mathematical Organization Theory 13(4), 407-422 (2007)

12. Yeh, C.C.M., et al.: Matrix profile i: all pairs similarity joins for time series: a unifying view that includes motifs, discords and shapelets. In: 2016 ICDM. pp. 1317-1322. IEEE (2016)

13. Zhou, C., et al.: Anomaly detection with robust deep autoencoders. In: Proceedings of the 23rd ACM SIGKDD. pp. 665-674. ACM (2017) 